\title{
Does Breastfeeding Have an Effect on Infant Mortality in India? An Analysis of National Family Health Survey Data
}

\author{
Nomita Chandhiok ${ }^{*}$, Lucky Singh², Kh. Jitenkumar Singh², Damodar Sahu², \\ Arvind Pandey ${ }^{2}$ \\ ${ }^{1}$ Indian Council of Medical Research, New Delhi, India \\ ${ }^{2}$ National Institute of Medical Statistics, New Delhi, India \\ Email: n chandhiok@hotmail.com, lucky.5bhu@gmail.com, jitensinghkh@gmail.com, \\ sahuicmr@gmail.com, arvindp.nims@gmail.com
}

Received 24 June 2015; accepted 13 September 2015; published 16 September 2015

Copyright (C) 2015 by authors and Scientific Research Publishing Inc.

This work is licensed under the Creative Commons Attribution International License (CC BY). http://creativecommons.org/licenses/by/4.0/

(c) (i)

Open Access

\begin{abstract}
Introduction: Sub-optimal infant and early childhood feeding practices in India contribute to the high burden of child under-nutrition and infant death. Objective: To study the effect of breastfeeding duration on the survival of infants along with other demographic, socioeconomic and service related factors and to compare the decadal changes in the association. Methods: A total of 36,754 and 26,782 births less than 3 years of age from two rounds of the National Family Health Survey (NFHS) carried out in India during 1992-1993 (NFHS-1), and 2005-2006 (NFHS-3) respectively comprised the sample. Infant mortality was examined against a set of variables and breastfeeding as a time dependent covariate using multivariate extended Cox regression model. Results: Hazard of infant death observed in NFHS-3 $(2005-2006)$ was 18 percent less $[\mathrm{HR}=0.82 ; 95 \% \mathrm{CI}=$ 0.759 - 0.879] as compared to that observed in NFHS-1 (1992-1993). After adjustment of other factors, the risk of infant death was $97 \%$ less amongst children who were breastfed [HR $=0.03 ; 95 \% \mathrm{CI}$ $=0.029-0.033$ ] as compared to those who were not breastfed. In contrast to rural area, babies belonging to urban area had an 18 percent less hazard of death during the infancy period [HR $=0.82$ ]. Hazard of infant death was less among births to mothers with higher maternal education levels $[H R=0.93]$ and in those who were in gainful occupation $[\mathrm{HR}=0.91]$. The risk of infant death was higher in babies perceived to be small at birth $[\mathrm{HR}=1.40]$ and belonging to states with medium post neonatal mortality rate (PNMR) $[\mathrm{HR}=1.23 ; 95 \% \mathrm{CI}=1.112-1.371]$ and high PNMR [HR = 1.46; $95 \% \mathrm{CI}=1.327-1.609]$ in contrast to those born to women belonging to states with low post neonatal mortality. Conclusion: Promotion and implementation of evidenced based strategies are needed in India to combat the high rates of child under-nutrition and infant and child mortality.
\end{abstract}

\footnotetext{
*Corresponding author.
}

How to cite this paper: Chandhiok, N., Singh, L., Singh, Kh.J., Sahu, D. and Pandey, A. (2015) Does Breastfeeding Have an Effect on Infant Mortality in India? An Analysis of National Family Health Survey Data. Open Journal of Preventive Medicine, 5, 359-369. http://dx.doi.org/10.4236/ojpm.2015.59040 


\section{Keywords}

\section{Breastfeeding, Infant Death, Factors, India}

\section{Introduction}

Sub-optimal breastfeeding is estimated to be responsible for 1.4 million child deaths and 43.5 million Disability Adjusted Life Years (DALYs) with non-exclusive breastfeeding accounting for 77 percent (1.06 million) of the deaths and 85 percent of the DALYs [1]. About one-fourth to one-half of all infant deaths in developing countries occur in the first week of life. Breastfeeding helps prevent hypothermia and hypoglycaemia in newborn babies, which are contributory causes of early neonatal deaths especially among low birth weight and premature babies. Immediate breastfeeding within the first hour, followed by early exclusive breastfeeding, improves the health and survival status of newborns. During the late neonatal period, most deaths in developing countries are due to infections such as sepsis, acute respiratory tract infection, meningitis, omphalitis and diarrhoea. Feeding colostrum and breastfeeding, especially exclusive breastfeeding, protect against such deaths. Among 23 interventions considered by the Bellagio Child Survival Study Group, scale-up of exclusive breast-feeding of infants for 6 months and continued feeding until 1 year could prevent an estimated 1.3 million child deaths per year [2].

Breastfeeding provides young infants with the nutrients they need for healthy growth and development. Apart from being a natural source of nourishment, human milk contains glycans and secretory immunoglobulin, which offer protection against infectious disease [3] [4] reducing the morbidity and mortality due to infectious diseases in childhood [5]. During the first six months of life, when digestive systems are not yet mature, exclusive breastfeeding offers additional protection from illness by limiting exposure to contaminated foods and liquids. This is particularly important in poor environments where early introduction of formula/animal milk is of particular concern because of the risk of pathogens, contamination and over dilution of milk leading to increased risks of morbidity and under-nutrition. A pooled analysis of studies carried out in middle/low income countries showed that breastfeeding substantially lowered the risk of death from infectious diseases in the first two years of life [6] and optimal breastfeeding practices could prevent a substantial proportion of hospital admissions due to diarrhea and lower respiratory tract infection [7]. A systematic review by Kramer et al. confirmed that exclusive breastfeeding in the first 6 months decreased morbidity from gastrointestinal and allergic diseases, without any negative effects on growth [8]. A pooled analysis of data from 3 countries indicated that either predominately or exclusively breastfed infants were at substantially lower risk for infant mortality than non-breastfed infants [9]. Over the past decades, available evidence for the health advantages of breastfeeding has continued to increase and these health benefits are seen to extend into adulthood [10]. Breastfeeding has protective roles against obesity, hypertension, dyslipidemia, and type II diabetes mellitus during adulthood that would have long-term beneficial health effects at individual and population levels [11]. Given such evidences, the WHO has recommended that every child should be exclusively breastfed for the first six months of life with partial breastfeeding continued until two years of age [12].

India is committed to achieving its National and Millennium Development Goal 4 of reduction in infant mortality. Recognition of breastfeeding as a core component of infant survival, growth and development has led to efforts that promote sound infant and young child nutrition. Guidelines towards this have been given by the Government of India [13] and initiatives to promote breastfeeding have been implemented through the national health programs. In India there are considerable differences in breastfeeding practices, access and utilization of health services and health outcomes across states/provinces and socio-economic groups [14]-[16]. Many studies have been carried out on determinants of infant mortality but very few have examined the trend and relationship between breastfeeding and infant death utilizing survey data of specific population [17]-[19]. This information would be important for evaluating the success of ongoing programs and various other activities that promote breastfeeding. This paper attempts to study the effect of breastfeeding duration on infant survival along with other demographic, socioeconomic and service related factors in India. It is based on the National Family Health Survey (NFHS) conducted with an aim to help the planners and policy makers for informed decision making, besides keeping a track of the health trends in the country. Furthermore, overall development and improved programme coverage in India have led to improvements in child survival. Hence, it may be desirable to examine 
changes related to breastfeeding and infant mortality over time. The study adopts a multivariate hazard model to analyze the determinants of infant mortality with duration of breastfeeding as a time dependent covariate using two rounds of the National Family Health Survey as well as by pooling the data to capture differences between surveys.

\section{Materials and Methods}

\subsection{Data Source}

India's National Family Health Survey (NFHS) provides state and national level information on fertility, family planning, infant and child morbidity and mortality, maternal and reproductive health, nutritional status of women and children, and on the quality of health services. The survey is similar to Demographic Health Survey (DHS) in other countries. It has adopted multistage stratified sampling methods to create a sample representing individuals from all 29 Indian states. So far three rounds of NFHS have been conducted in the year 1992-1993 (NFHS-1), 1998-1999 (NFHS-2) and 2005-2006 (NFHS-3). The present study uses data from the NFHS-1 and NFHS-3 keeping pace for the changes in the study variable as well as explanatory factors.

To make the breastfeeding estimates representative and comparable across the two survey rounds, we used appropriate sample weights in the analysis. Sampling design, sample size and response rate details are published in the round-specific survey reports [20] [21]. All eligible women aged 15 - 49 years were asked to provide information on complete birth history including sex, month and year of birth, and survival status for each live birth. The information on age at death was recorded in days for children who had died in the first month of life; in months for children who had died after the first month but before completion of their second birthday, and in years for children who had died at later ages. For children who had died after their second birthday, age at death in months was computed. Detailed information on antenatal, delivery and postnatal care for births which occurred to eligible women four and five years preceding the survey in NFHS-1 and NFHS-3 respectively were collected. The present study was restricted to all live births that occurred three years preceding survey for analysis of infant mortality. For the purpose of this study, the north-eastern states were excluded and only 18 states and the National capital region of Delhi were considered for the analysis. A total of 36754 births in NFHS- 1 and 26782 births in NFHS-3 were included in the analysis.

\subsection{Variable Definition}

Dependent variable: Dependent variable is infant mortality which is defined as the probability of dying before the first birthday.

Independent variables: The following socio-economic, demographic and health services access variables were considered: breastfeeding (no/yes); place of residence (rural/urban); mother's age at the time of child birth (15 19, 20 - 34, 35+ years); mothers education (illiterate, literate but below middle class, middle class and above); mother's occupation (non gainful occupation, gainful occupation); preceding birth interval ( $<2$ years, $\geq 2$ years); antenatal and natal care (no/yes); size of the child at birth (small, medium/large), standard of living index based on ownership of household goods (low, medium/high). In the absence of direct information on use of health care services proxy measures such as number of antenatal check-ups, number of tetanus toxoid injections (TT) received by mother and place of delivery were used as indicators of access to use of health care facilities. A "variable antenatal and natal care” was created. if mother received at least three antenatal check-ups during pregnancy or received two doses of TT immunization or delivered the child in a health facility then antenatal and natal care received by the mother was coded as "yes", otherwise, coded as "no". States were stratified on the basis of post neonatal mortality rate (low mortality states, medium/high mortality states) as observed in Sample Registration System (SRS) [22].

\subsection{Statistical Analysis}

Extended Cox regression model has been used to examine the effect of socio-economic, demographic and health service access factors on risk of infant mortality. An extended Cox Regression model, which allows us to specify time-dependent covariates, can be used in situations where the proportional hazards assumption of the Cox Regression model does not hold [23], i.e., hazard ratios change across time; the values of one (or more) of the covariates are different at different time points. The general hazard ratio formula for extended Cox model is 
shown below:

$$
\widehat{H R}(t)=\frac{\hat{h}\left(t, X^{*}(t)\right)}{\hat{h}(t, X(t))}=\exp \left[\sum_{i=1}^{p 1} \hat{\beta}_{i}\left[X_{i}^{*}-X_{i}\right]+\sum_{j=1}^{p 2} \hat{\delta}_{j}\left[X_{j}^{*}(t)-X_{j}(t)\right]\right]
$$

where,

$X_{1}, X_{2}, \cdots, X_{p 1}$ are fixed variables and $X_{1}(t), X_{2}(t), \cdots, X_{p 2}(t)$ are time-dependent variables, and $\beta$ and $\delta$ denote vector of coefficients of the respective explanatory variables.

For this model, we have defined dependent variable as time (age of the child) and survival status of child (1 if event is occurred, i.e., child died and 0 if child is alive) during period of infant ( $<12$ months). Results of the multivariate analyses are presented as hazard ratios (risk of dying). The hazard ratio (HR) is the relative increase or decrease in the risk of dying associated with the specific category of predictor variable when compared to the reference category for that variable. If hazard ratio $>1$ for a predictor variable, it means that the hazard is greater, i.e., increased risk of death and if hazard ratio $<1$, implies a decreased risk of death.

The statistical analysis was conducted using SPSS version 19 using complex survey design. All tests were two-sided and $\mathrm{p}<0.05$ was considered statistically significant.

\subsection{Ethical Considerations}

All study procedures, consent forms and tools were approved by the International Institute of Population Science Ethics Review Board (IIPS, 1992-1993, IIPS 2005-2006).

\section{Results}

Unadjusted hazard ratio depicting associations between infant mortality and socio-economic and demographic factors are presented in Table 1. Breastfeeding, place of residence, mothers age at birth, education, mother in gainful occupation, birth interval, antenatal/natal care, size of the baby and standard of living Index and stratification of states by level of PNMR (post neonatal mortality rate) were found to be significantly associated with infant death during 1992-1993 (NFHS-1), 2005-2006 (NFHS-3) as well as during 1992-2006.

The unadjusted hazard ratio for infant deaths in NFHS-1 (1992-1993) divulged that the risk of infant death was 97 percent $[\mathrm{HR}=0.030,95 \% \mathrm{CI}=0.028-0.033]$ less among those children who were breastfed as compared to those who were not breastfed. In contrast to rural area, babies belonging to urban area had a 34 percent [95\% CI $=0.612-0.707]$ less hazard of death during the infancy period. Hazard of infant death was higher among births to mothers aged $15-19$ years $(\mathrm{HR}=1.41)$ and aged $35+(\mathrm{HR}=1.47)$ as compared with births to the reference mother's age at child birth of 20 - 34 years. Children born to mothers having less than 8 years of education or 8 years complete and above had 27 percent and 52 percent less hazard of infant death than children born to illiterate women respectively. Babies born to mothers who were in gainful occupation had a 6 percent less hazard of infant death than babies born to mothers who were not gainfully occupied. The hazard of infant death was 36 percent higher when the birth interval was less than 24 months as compared to a longer birth interval of 24 months or more. Children' mothers who received antenatal/natal care, the hazard of infant mortality was estimated at 0.49 [95\% CI $=0.461$ - 0.528] which were substantially lower than that seen in women who did not receive it. Small size of baby at birth had an increased infant death risk of 2.5 times when compared with a large or medium size baby [p value $=0.000$ ]. The risk of infant dying was 35 percent less among those with a high standard of living index. A higher hazard of infant mortality was observed in babies born to women belonging to medium PNMR (48\%) and high PNMR (2 times) states in contrast to those born to women belonging to states with low PNMR.

The unadjusted hazard ratio for infant deaths in NFHS-3 (2005-2006) showed that the likelihood of infant death was lower among the children who were breastfed as compared to those who were not breastfeed [HR = 0.023; 95\% CI $=0.021-0.026]$. The risk of infant dying was 33 percent less among urban children than the rural children [p value $=0.00$ ]. Babies born to mothers aged $15-19$ years and aged 35+ were 46 percent $[95 \% \mathrm{CI}=$ 1.327 - 1.595] and 28 percent [95\% CI $=1.056$ - 1.552] higher hazard of infant death than babies born to mothers 20 - 34 years of age respectively. Children with mothers having 8 years or more than 8 years of education had an decreases infant death risk of 48 percent when compared with children with illiterate mothers [p value $=0.000$ ]. However children with mother having less than 8 years of education were 14 percent [ $95 \% \mathrm{CI}=0.778-0.948$ ] 


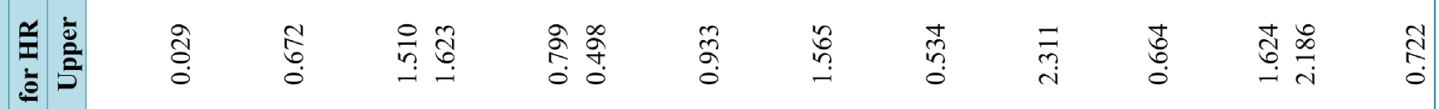

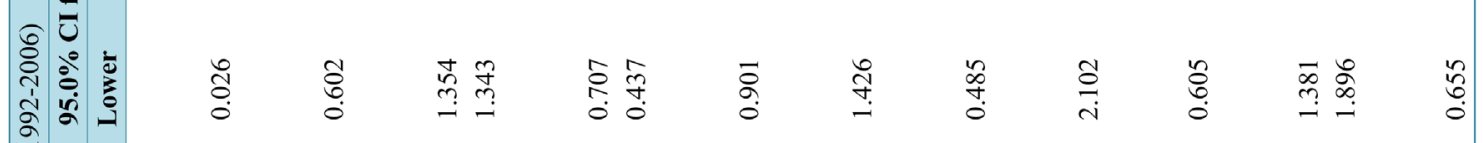

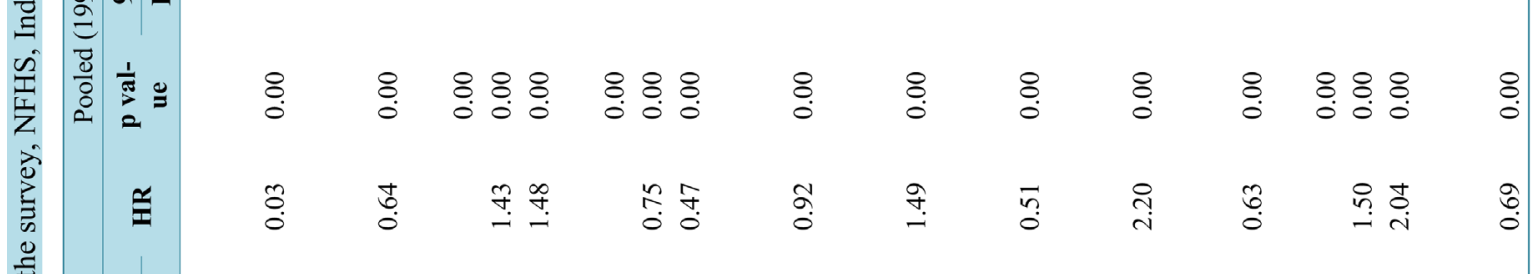

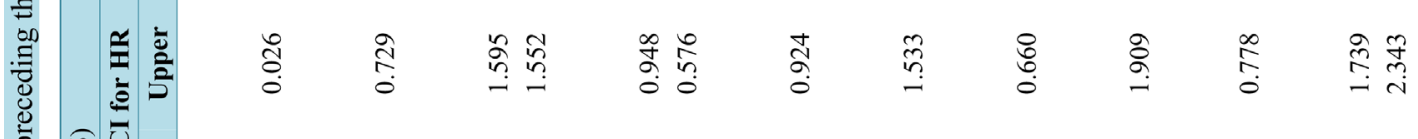

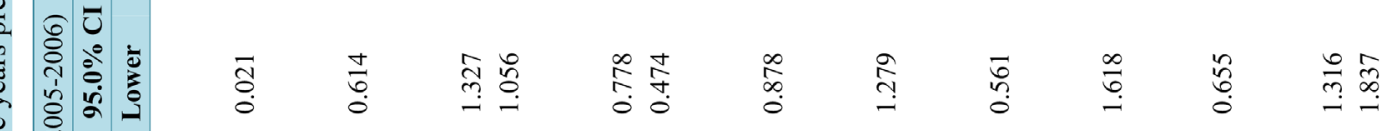

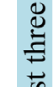

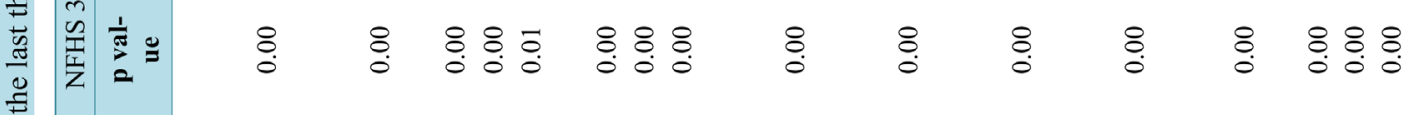

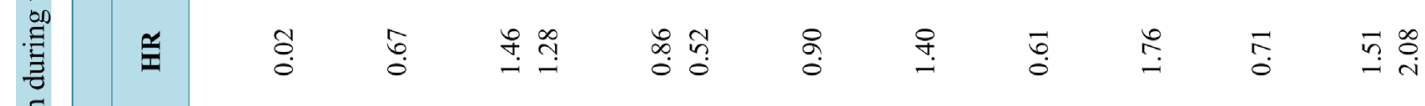

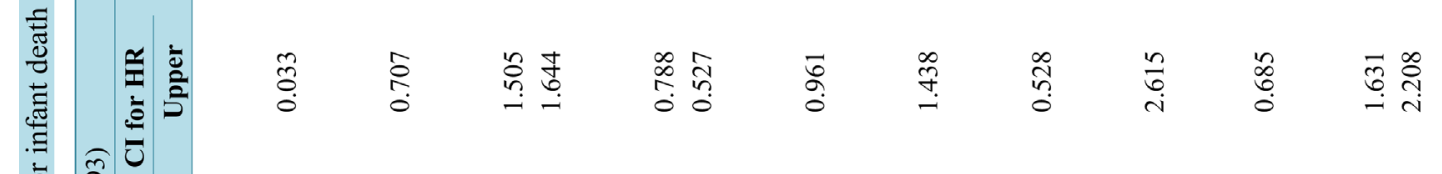

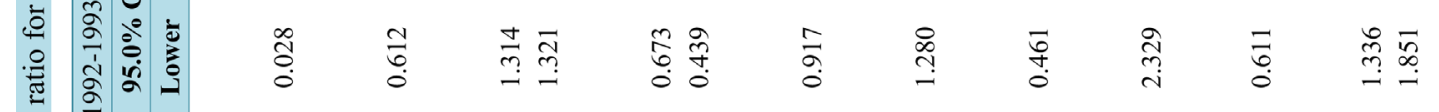

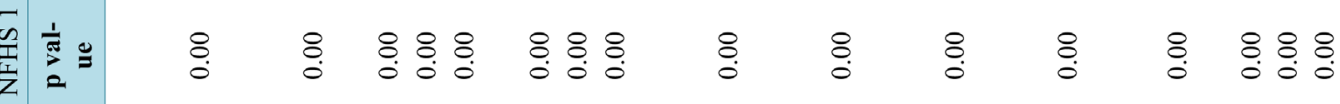

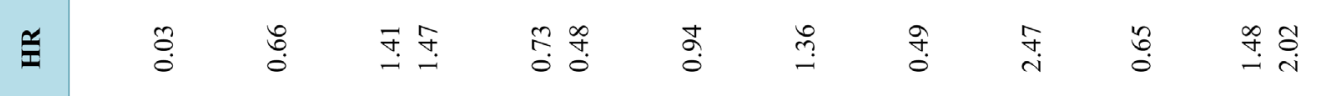

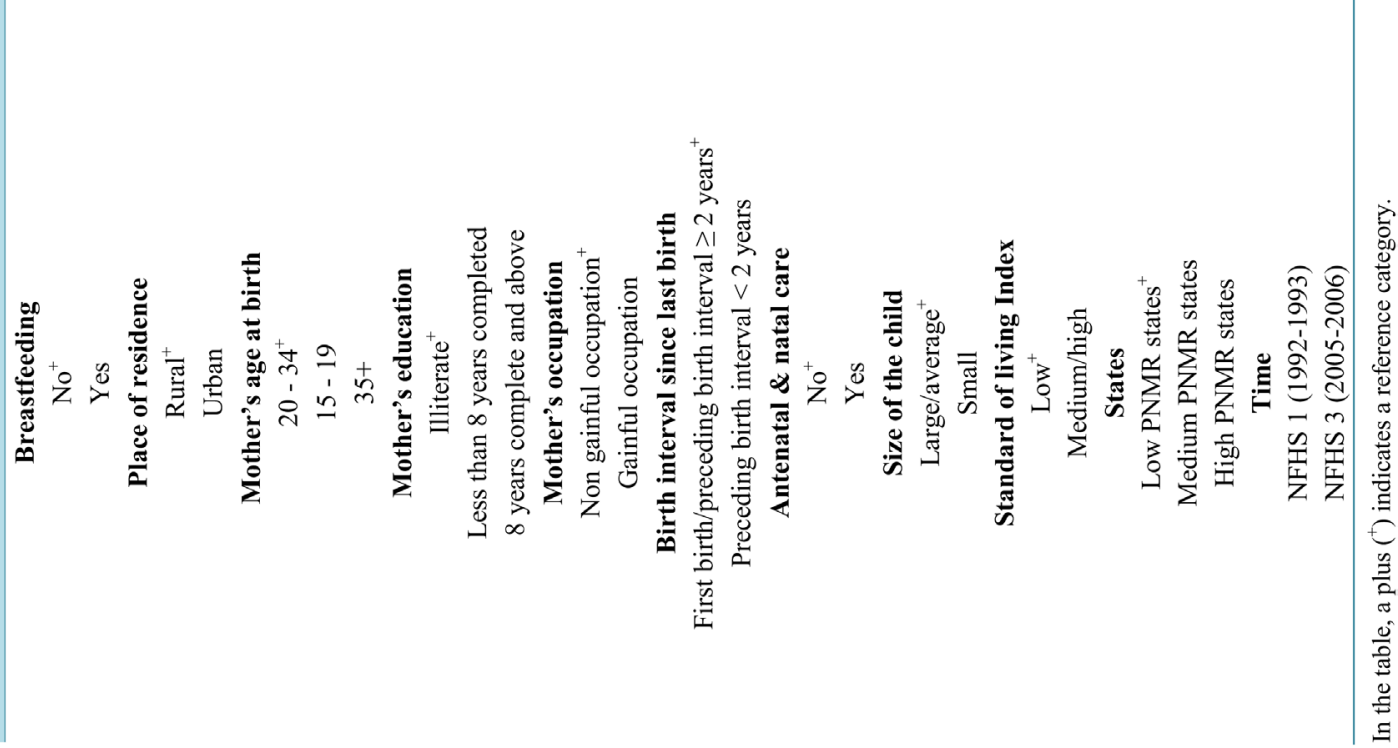


less hazard of infant death than the children with illiterate mothers. In children whose mothers were engaged in gainful occupation, the hazard of infant mortality was estimated at 0.90 [95\% CI $=0.88-0.92]$ which were substantially lower than that seen in children of mothers who were not engaged in gainful occupation. The hazard of infant death was 40 percent [95\% CI $=1.28$ - 1.53] higher when the birth interval was less than 24 months as compared to a longer birth interval of 24 months or more. The risk of infant dying was 39 percent $[95 \% \mathrm{CI}=$ 0.56 - 0.66] less among children whose mothers received antenatal care as compared to those whose mothers did not receive antenatal care. Small size babies were having 76 percent higher risk of infant death than large/ medium size babies $(\mathrm{p}=0.000)$. Babies belonging to wealthiest household were 29 percent less hazard of infant death than Babies belonging to poor household $(\mathrm{p}=0.000)$. In contrast to low PNMR states, babies belonging medium and high PNMR states had 51 percent [95\% CI $=1.32-1.74$ ] and 2.8 times [95\% CI $=1.84-2.34$ ] higher hazard of death during the infancy period.

Unadjusted pooled sample (NFHS-1 and NFHS-3) analysis indicates that there was significant decline in infant death between NFHS-1 and NFHS-3. The infant mortality observed in NFHS-3 was 31 percent less as compared to that observed in NFHS-1 [HR $=0.69 ; 95 \% \mathrm{CI}=0.66-0.72]$. The risk of infant dying was 97 percent less among those children who were breastfed as compared to those who were not breastfed $[\mathrm{p}$ value $=$ 0.00]. In contrast to rural area, babies belonging to urban area had a 36 percent less hazard of death during the infancy period [p value $=0.00$ ]. Hazard of infant mortality was higher among births to mothers aged $15-19$ years $[\mathrm{HR}=1.43 ; 95 \% \mathrm{CI}=1.354-1.510]$ and mothers aged $35+[\mathrm{HR}=1.48 ; 95 \% \mathrm{CI}=1.343-1.623]$ as compared with births to the reference mother's age at child birth of $20-34$ years. Education of the mother was inversely associated with infant death ( $p$ value $=0.00$ ). Women who were in gainful occupation had an 8 percent less hazard of infant death than women who were not gainfully occupied [p value $=0.00$ ]. The hazard of infant mortality was 49 percent higher when the birth interval was less than 24 months as compared to a longer birth interval of 24 months or more [HR $=1.49$; 95\% CI $=1.426-1.565]$. Among women who received antenat$\mathrm{al} /$ natal care, the hazard of infant mortality was estimated at 0.51 [95\% CI $=0.485-534]$ which were substantially lower than that seen in women who did not receive it.

Small size baby had an increased infant mortality risk of 2 times at birth when compared with a large or medium size baby [p value $=0.00$ ]. The risk of infant dying was 37 percent less among those with a high standard of living index compare with low standard of living. A greater hazard of infant mortality was observed among babies born to women belonging to states which have medium to high post neonatal mortality states in contrast to those born to women belonging to states with low PNMR.

The results under extended Cox proportional hazard multivariate model reveal that the hazard of infant death in NFHS-3 (2005-2006) is 18 percent less [95\% CI $=0.759$ - 0.879] as compared to that in NFHS-1 (1992-1993). The risk of infant death is 97 percent less [95\% CI $=0.029$ - 0.033] among those children who were breastfed as compared to those who were not breastfed. In contrast to rural area, babies belonging to urban area had an 18 percent less hazard of death during the infancy period [p value $=0.00$ ]. Hazard of infant death was less in births among educated mothers (8 or more years of education) $[\mathrm{HR}=0.66$; $95 \% \mathrm{CI}=0.600-0.728]$ as compared with births in illiterate mothers after controlling for other socio-economic and demographic variables. The births to women who were in gainful occupation had a 9 percent less hazard of infant death than those borne to women who were not gainfully occupied ( $\mathrm{p}$ value $=0.00$ ). When size of baby at birth was considered, small size baby had an increased risk of infant death (40 percent) when compared with a large or medium size baby. A greater hazard of infant death was observed in babies born to women from states/provinces which had medium post neonatal mortality [HR $=1.23$; 95\% CI $=1.112-1.371]$ and high post neonatal mortality $[\mathrm{HR}=1.46 ; 95 \% \mathrm{CI}=$ 1.327 - 1.609] in contrast to those born to women belonging to states/provinces with low post neonatal mortality (Table 2).

\section{Discussion}

This paper, using data from two rounds of India's National Family Health Survey, i.e., NFHS-1 and NFHS-3 that were carried out more than 10 years apart, provides evidence on the trends and association of breastfeeding with infant survival in India's states/provinces stratified on the basis of post neonatal mortality. As mentioned in the method section, we did not include data from NFHS-2 as appreciable changes in determinants of infant mortality may not be expected in the five year that elapsed between successive surveys. Post neonatal mortality was used as a basis for stratification as socio-economic, programmatic and development efforts are reported to have strong effects on post neonatal mortality than on neonatal mortality. 
Table 2. Cox proportion hazard regression model showing Adjusted hazard ratio for infant death during the last three years preceding the survey, NFHS, India.

\begin{tabular}{|c|c|c|c|c|}
\hline \multirow{3}{*}{ Variables } & \multicolumn{4}{|c|}{ Pooled (1992-2006) } \\
\hline & \multirow{2}{*}{ HR } & \multirow{2}{*}{ p value } & \multicolumn{2}{|c|}{$95.0 \%$ CI for HR } \\
\hline & & & Lower & Upper \\
\hline \multicolumn{5}{|l|}{ Breastfeeding } \\
\hline \multicolumn{5}{|l|}{$\mathrm{No}^{+}$} \\
\hline Yes & 0.03 & 0.00 & 0.029 & 0.033 \\
\hline \multicolumn{5}{|l|}{ Place of residence } \\
\hline \multicolumn{5}{|l|}{ Rural $^{+}$} \\
\hline Urban & 0.82 & 0.00 & 0.762 & 0.887 \\
\hline \multicolumn{5}{|l|}{ Mothers age at birth } \\
\hline $20-34^{+}$ & & 0.07 & & \\
\hline $15-19$ & 1.07 & 0.08 & 0.993 & 1.153 \\
\hline $35+$ & 1.10 & 0.09 & 0.986 & 1.225 \\
\hline \multicolumn{5}{|l|}{ Mother's education } \\
\hline Illiterate $^{+}$ & & 0.00 & & \\
\hline Less than 8 years completed & 0.93 & 0.08 & 0.856 & 1.009 \\
\hline 8 years complete and above & 0.66 & 0.00 & 0.600 & 0.728 \\
\hline \multicolumn{5}{|l|}{ Mother's occupation } \\
\hline \multicolumn{5}{|l|}{ Non gainful occupation $^{+}$} \\
\hline Gainful occupation & 0.91 & 0.00 & 0.892 & 0.926 \\
\hline \multicolumn{5}{|l|}{ Birth interval since last birth } \\
\hline \multicolumn{5}{|l|}{ First birth/preceding birth interval $\geq 2$ years $^{+}$} \\
\hline Preceding birth interval $<2$ years & 1.06 & 0.08 & 0.993 & 1.130 \\
\hline \multicolumn{5}{|l|}{ Antenatal \& natal care } \\
\hline \multicolumn{5}{|l|}{$\mathrm{No}^{+}$} \\
\hline Yes & 1.03 & 0.44 & 0.956 & 1.109 \\
\hline \multicolumn{5}{|l|}{ Size of the child } \\
\hline \multicolumn{5}{|l|}{ Large/average $^{+}$} \\
\hline Small & 1.40 & 0.00 & 1.322 & 1.493 \\
\hline \multicolumn{5}{|l|}{ Standard of living Index } \\
\hline \multicolumn{5}{|l|}{ Low $^{+}$} \\
\hline Medium/high & 0.96 & 0.21 & 0.894 & 1.025 \\
\hline \multicolumn{5}{|l|}{ States } \\
\hline Low PNMR states ${ }^{+}$ & & 0.00 & & \\
\hline Medium PNMR states & 1.23 & 0.00 & 1.112 & 1.371 \\
\hline High PNMR states & 1.46 & 0.00 & 1.327 & 1.609 \\
\hline \multicolumn{5}{|l|}{ Time } \\
\hline \multicolumn{5}{|l|}{ NFHS 1 (1992-1993) } \\
\hline NFHS 3 (2005-2006) & 0.82 & 0.00 & 0.759 & 0.879 \\
\hline
\end{tabular}

In the table, a plus $\left(^{+}\right)$indicates a reference category. 
Over the years, several initiatives have been taken by the Government of India in its efforts to achieve its National and Millennium Development Goal of reduction in infant mortality [24]. Our study indicates that these efforts have led to some decline in the proportion of infant deaths which is also in accordance with decline observed in the infant mortality rate over time [25].

The effects of social, economic, cultural and geographical variables are said to exert an impact on mortality and Mosley and Chen identified a set of 14 intermediate variables which directly influence the risk of morbidity and mortality [26]. For the present study, we have considered the effect of only socio-economic, demographic, any breastfeeding and health delivery access variables on infant death. Our analysis revealed that children who were not breastfed, belonged to rural area, whose mothers were illiterate, not gainfully employed or were not in the prime reproductive age of 20 - 34 years, with a preceding birth interval of less than 24 months, did not receive antenatal care during pregnancy or natal care during delivery, were perceived by their mothers to be smaller at birth, were from a low SLI and belonged to a high post neonatal mortality State/province experienced a greater risk of dying in both the time periods. This indicates that the relevance and association of these factors to infant death is still significant. Research in other developing countries has demonstrated findings in consonance to our study [27] [28]. Addressing these factors through well planned programs could lead to reduction in infant deaths.

To obtain the effect of each factor independent of the other, hazard regression model was used as many of the mortality factors were correlated. Besides mothers' age at birth, birth spacing since last birth, availing health services like antenatal and natal care and wealth index, all other factors studied showed an independent bearing on infant survival. Even though there was a strong inverse association between education and infant mortality at the bivariate (unadjusted) level, this effect was diminished after adjusting for other variables included in the model suggesting its possible association with other factors and hazard of infant mortality was only seen to be less among births to mothers who were better educated ( $>8$ years of education).

It is well documented that breastfeeding is the unique source of nutrition and plays an important role in the growth, development and survival of infants. A strong association of breastfeeding and infant mortality was observed over both the time periods. The risk of infant dying was 97 percent less among those children who were breastfed as compared to those who were not breastfed in the unadjusted pooled sample analysis and after adjustment of other factors. A number of studies have found the positive effect of breastfeeding on infant survival [17]. The relationship is found to be stronger among children who come from poor families, whose mothers have little education and whose family lack access to safe drinking water and sanitary toilet facilities. Some reports have documented that in communities with high prevalence of malnutrition and poor sanitation, breastfeeding substantially enhances the child survival up to three years of age. Also, the protective effect of breastfeeding tends to be greater for younger infants than for older infants. The benefits of breastfeeding in combination with supplementary food are found to continue well beyond the first year of life particularly in developing countries. In a multi-country study, non-breastfed infants had a higher risk of dying when compared with those who had been predominantly breastfed $(\mathrm{HR}=10.5 ; 95 \% \mathrm{CI}=5.0-22.0$; $\mathrm{p}<0.001)$ as did partially breastfed infants $(\mathrm{HR}=2.46 ; 95 \% \mathrm{CI}=1.44-4.18 ; \mathrm{p}=0.001)$ [8]. In a study carried out by Betran, attributable fraction analysis of national data on infant mortality and breastfeeding was carried out from Latin America and the Caribbean. The study concluded that promotion of breastfeeding has an important role in increasing survival of infants [18].

A few studies have attempted to identify factors associated with sub-optimal breastfeeding of infants in India. Chudasama et al. (2009) reported parity, delivery interval more than 24 months, early maternal age, lower socio-economic status, low paternal education and occupation as a risk factor for early weaning [29]. Breastfeeding prevalence was higher among illiterate mothers and mothers belonging to lower socio-economic status [30]. Patel et al. (2010) analyzing NFHS-3 data found lower rates of exclusive breastfeeding in children of women from higher quintiles of wealth (adjusted OR for rich $=1.71$; 95\% CI, 1.25 to 2.33; adjusted OR for richest $=$ 2.03, 95\% CI, 1.40 to 2.94) and those who delivered in a health facility (adjusted OR $=1.35$; $95 \%$ CI, 1.06 to 1.71). The negative association could be due to greater affordability in higher wealth index households to foods other than breast milk [31]. Antenatal contact with a health professional provides an opportunity to counsel a woman to establish and continue EBF and lack of breastfeeding counselling was significantly associated with decreased rates of EBF at 4 and 6 months in rural north India [32].

The large sample size, country wide representation and effect of factors correlated with infant death at two time points more than a decade apart contributes to the strength of the study. The evidence on the health conse- 
quences of breastfeeding is mostly based on observational studies because of the ethical challenges in carrying out randomized trials. Our study limitation is that it utilizes health survey data and is hence limited by the survey design and information collected. It does not capture the information on childcare practices such as exclusive breastfeeding of the children who died. Besides, cross-sectional observational studies of breastfeeding and infant death may be affected by a host of methodological problems like self-selection, confounding and reverse causality [33]-[35]. The confounding in our study was addressed by adjusting for most of the other important determinants known to be associated with infant mortality or breastfeeding. Inaccurate measurement of breastfeeding exposure or outcome may lead to differential or non-differential misclassification. Since we only used current status data this was obviated.

\section{Conclusion}

India has been facing high rates of both child under-nutrition and infant and child mortality requiring evidence based strategies to address the situation. Promotion and implementation of exclusive breastfeeding are the single most effective intervention for child survival [36] [37]. To universalize optimal infant feeding practices is a serious challenge and would require a great deal of planned action. Though policies and programs for this have been put in place, their implementation is critical to prevent a plateau in reduction of infant deaths.

\section{Acknowledgements}

Authors would like to thank the National Institute of Medical Statistics for support given in analyzing the data. This study is part of a PhD thesis work under GGS IP University and we whole heartedly thank them for allowing us to publish this work.

\section{Authors' Contribution}

NC, JS and AP contributed in the concept of the analysis of NFHS dataset. JS, LS and NC performed statistical analysis. NC wrote manuscript with significant contribution from AP. AP and DS supervised the analysis and interpretation of the results. All authors have read and approved the final manuscript.

\section{Competing Interests}

The authors declare that they have no competing interests.

\section{References}

[1] Black, R.E., Allen, L.H., Bhutta, Z.A., Caulfield, L.E., de Onis, M., Ezzati, M., Mathers, C. and Rivera, J. (2008) Maternal and Child Undernutrition Study Group. Maternal and Child Undernutrition: Global and Regional Exposures and Health Consequences. Lancet, 371, 243-260. http://dx.doi.org/10.1016/S0140-6736(07)61690-0

[2] Jones, G., Steketee, R.W., Black, R.E., Bhutta, Z.A. and Morris, S.S., Bellagio Child Survival Study Group (2003) How Many Child Deaths Can We Prevent This Year? Lancet, 362, 65-71. http://dx.doi.org/10.1016/S0140-6736(03)13811-1

[3] Morrow, A.L., Ruiz-Palacios, G.M., Jiang, X. and Newburg, D.S. (2005) Human-Milk Glycans That Inhibit Pathogen Binding Protect Breast-Feeding Infants against Infectious Diarrhea. Journal of Nutrition, 135, 1304-1307.

[4] Noguera-Obenza, M., Ochoa, T.J., Gomez, H.F., Guerrero, M.L., Herrera-Insua, I., Morrow, A.L., Ruiz-Palacios, G., Pickering, L.K., Guzman, C.A. and Cleary, T.G. (2003) Human Milk Secretory Antibodies against Attaching and Effacing Escherichia coli Antigens. Emerging Infectious Diseases, 9, 545-551. http://dx.doi.org/10.3201/eid0905.020441

[5] (2013) Short-Term Effects of Breastfeeding: A Systematic Review on the Benefits of Breastfeeding on Diarrhoea and Pneumonia Mortality. WHO, NLM Classification: WS 125.

[6] (2000) Effect of Breastfeeding on Infant and Child Mortality Due to Infectious Diseases in Less Developed Countries: A Pooled Analysis. WHO Collaborative Study Team on the Role of Breastfeeding on the Prevention of Infant Mortality. Lancet, 355, 451-455. http://dx.doi.org/10.1016/S0140-6736(99)06260-1

[7] Quigley, M.A., Kelly, Y.J. and Sacker, A. (2007) Breastfeeding and Hospitalization for Diarrheal and Respiratory Infection in the United Kingdom Millennium Cohort Study. Pediatrics, 119, e837-e842. http://dx.doi.org/10.1542/peds.2006-2256

[8] Kramer, M.S. and Kakuma, R. (2004) The Optimal Duration of Exclusive Breastfeeding: A Systematic Review. Ad- 
vances in Experimental Medicine and Biology, 554, 63-77. http://dx.doi.org/10.1007/978-1-4757-4242-8_7

[9] Bahl, R., Frost, C., Kirkwood, B.R., Edmond, K., Martines, J., Bhandari, N. and Arthur, P. (2005) Infant Feeding Patterns and Risks of Death and Hospitalization in the First Half of Infancy: Multicentre Cohort Study. Bulletin of the World Health Organization, 83, 418-426.

[10] Horta, B.L., Bahl, R., Martinés, J.C. and Victora, C.G. (2007) Evidence on the Long Term Effects of Breastfeeding. Systematic Reviews and Meta-Analysis. World Health Organization.

[11] Kelishadi, R. and Farajian, S. (2014) The Protective Effects of Breastfeeding on Chronic Non-Communicable Diseases in Adulthood: A Review of Evidence. Advanced Biomedical Research, 3, 3. http://dx.doi.org/10.4103/2277-9175.124629

[12] World Health Organization (2001) Global Strategy for Infant and Young Child Feeding. The Optimal Duration of Exclusive Breastfeeding. World Health Organization, Geneva.

[13] Ministry of Women and Child Development, Government of India. National Guidelines on IYCF, 2004. http://wcd.nic.in/nationalguidelines.pdf

[14] Dwivedi, L.K. (2010) Breastfeeding and Its Demographic and Health Implications in India. PhD Dissertation, International Institute for Population Sciences, Mumbai.

[15] Joe, W., Mishra, U.S. and Navaneetham, K. (2010) Socio-Economic Inequalities in Child Health: Recent Evidence from India. Global Public Health, 5, 493-508.

[16] Sen, G., George, A. and Stalin, P. (2002) Engendering International Health: The Challenge of Equity. MIT Press, Cambridge.

[17] Huffman, S.L., Zehner, E.R. and Victora, C. (2001) Can Improvements in Breast-Feeding Practices Reduce Neonatal Mortality in Developing Countries? Midwifery, 17, 80-92. http://dx.doi.org/10.1054/midw.2001.0253

[18] Betrán, A.P., de Onís, M., Lauer, J.A. and Villar, J. (2001) Ecological Study of Effect of Breast Feeding on Infant Mortality in Latin America. BMJ, 323, 303-306. http://dx.doi.org/10.1136/bmj.323.7308.303

[19] Habicht, J.P., DaVanzo, J. and Buzz, W.P. (1986) Does Breastfeeding Really Save Lives or Are the Potential Benefits Due to Biases? American Journal of Epidemiology, 123, 279-290.

[20] International Institute for Population Sciences (IIPS) (1995) National Family Health Survey (MCH and Family Planning), India 1992-93 (NFHS-1). IIPS, Bombay.

[21] International Institute for Population Sciences: Mumbai, India and ORC Macro; Maryland, USA. 2000. National Family Health Survey, India, 2005-2006 (NFHS-3). IIPS, Mumbai.

[22] Registrar General of India (1994) Sample Registration System: Fertility and Mortality Indicators 1992, Fertility and Mortality (SRS) Survey Division, Ministry of Home Affairs, New Delhi.

[23] Kleinbaum, D.G. and Klein, M. (2005) Survival Analysis: A Self-Learning Text. 2nd Edition, Springer, New York.

[24] (2015) Child Health Programme in India. http://mohfw.nic.in/WriteReadData/1892s/6342515027file14.pdf

[25] Reddy, H., Pradhan, M.R., Ghosh, R. and Khan, A.G. (2012) India’s Progress towards the Millennium Development Goals 4 and 5 on Infant and Maternal Mortality. WHO South-East Asia Journal of Public Health, 1, 279-289.

[26] Mosley, W.H. and Chen, L. (1984) An Analytical Framework for the Study of Child Survival in Developing Countries. In: Mosley, W.H. and Chen, L., Eds., Child Survival: Strategies for Research. Population and Development Review, 10, 25-45. http://dx.doi.org/10.2307/2807954

[27] Geib, L.T., Freu, C.M., Brandao, M. and Nunes, M.L. (2010) Social and Biological Determinants of Infant Mortality in Population Cohort in the City of Passo Fundo, Rio Grande do Sul State. Ciência \& Saúde Coletiva, 15, 363-370. http://dx.doi.org/10.1590/S1413-81232010000200011

[28] Haroun, H.M., Mahfouz, M.S. and Ibrahim, K.H. (2007) Level and Determinants of Infant and Under-Five Mortality in Wad-Medani Town, Sudan. Journal of Family and Community Medicine, 14, 65-69.

[29] Chudasama, R.K., Patel, P.C. and Kavishwar, A.B. (2009) Determinants of Exclusive Breastfeeding in South Gujarat Region of India. Journal of Clinical Medicine Research, 1, 102-108. http://dx.doi.org/10.4021/jocmr2009.06.1242

[30] Kumar, S., Nath, L.M. and Reddaiah, V.P. (1989) Factors Influencing Prevalence of Breastfeeding in a Resettlement Colony of New Delhi. The Indian Journal of Pediatrics, 56, 385-391. http://dx.doi.org/10.1007/BF02722307

[31] Patel, A., Badhoniya, N., Khadse, S., Senarath, U., Agho, K.E. and Michael, J. (2010) Infant and Young Child Feeding Indicators and Determinants of Poor Feeding Practices in India: Secondary Data Analysis of National Family Health Survey 2005-06. Food and Nutrition Bulletin, 31, 314-333.

[32] Kishore, M.S.S., Kumar, P. and Aggarwal, A.K. (2009) Breastfeeding Knowledge and Practices amongst Mothers in a Rural Population of North India: A Community Based Study. Journal of Tropical Pediatrics, 55, 183-188. http://dx.doi.org/10.1093/tropej/fmn110 
[33] Sauls, H.S. (1979) Potential Effect of Demographic and Other Variables in Studies Comparing Morbidity of BreastFed and Bottle-Fed Infants. Pediatrics, 64, 523-527.

[34] Victoria, C.G. (1990) Case-Control Studies on the Influence of Breastfeeding on Child Morbidity and Mortality: Methodological Issues. In: Atkinson, S.A., Hanson, L.A. and Chandra, R.K., Eds., Breastfeeding, Nutrition, Infection and Infant Growth in Developed and Developing Countries, Arts Biomedical, St. John's, 405-418.

[35] Bhutta, Z.A., Ahmed, T., Black, R.E., Cousens, S., Dewey, K., Giugliani, E., Haider, B.A., Kirkwood, B., Morris, S.S., Sachdev, H.P. and Shekar, M. (2008) Maternal and Child Undernutrition Study Group. What Works? Interventions for Maternal and Child Undernutrition and Survival. Lancet, 371, 417-440. http://dx.doi.org/10.1016/S0140-6736(07)61693-6

[36] Darmstadt, G.L., Bhutta, Z.A., Cousens, S., Adam, T., Walker, N. and de Bernis, L. (2005) Lancet Neonatal Survival Steering Team. Evidence-Based, Cost-Effective Interventions: How Many Newborn Babies Can We Save? Lancet, 365, 977-988. http://dx.doi.org/10.1016/S0140-6736(05)71088-6

[37] Gupta, A., Dadhich, J.P. and Faridi, M.M. (2010) Breastfeeding and Complementary Feeding as a Public Health Intervention for Child Survival in India. Indian Journal of Pediatrics, 77, 413-418. http://dx.doi.org/10.1007/s12098-010-0049-6

\section{Abbreviations}

NFHS-1: National Family Health Survey-1 (1992-1993);

NFHS-3: National Family Health Survey-3 (2005-2006);

DALYs: Disability Adjusted Life Years;

(DHS): Demographic Health Survey;

IIPS: Indian Institute of Population Studies;

TT: Tetnus Toxoid;

HR: Hazard Ratio;

SLI: Standard of Living Index;

WHO: World Health Organization;

PNMR: Post Neonatal Mortality Rate. 